\section{PSICOLOGIA IBEROAMERICANA}

\section{Psicología lberoamericana}

ISSN: 1405-0943

psicología.iberoamericana@uia.mx

Universidad Iberoamericana, Ciudad de

México

México

García Meraz, Melissa; Salvador García, Alejandra; Guzmán Saldaña, Rebeca Ma. Elena Actitudes hacia la transformación de la vida en pareja: soltería, matrimonio y unión libre

Psicología Iberoamericana, vol. 20, núm. 2, julio-diciembre, 2012, pp. 16-25

Universidad Iberoamericana, Ciudad de México

Distrito Federal, México

Disponible en: http://www.redalyc.org/articulo.oa?id=133928816003

Cómo citar el artículo

Número completo

- Más información del artículo

- Página de la revista en redalyc.org

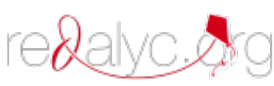

Sistema de Información Científica

Red de Revistas Científicas de América Latina, el Caribe, España y Portugal

Proyecto académico sin fines de lucro, desarrollado bajo la iniciativa de acceso abierto 


\title{
Actitudes hacia la transformación de la vida en pareja: soltería, matrimonio y unión libre
}

\author{
Attitudes towards Transformation of Couple Live: Singlehood, \\ Marriage and Cohabitation
}

\author{
Melissa García Meraz* \\ Alejandra Salvador García** \\ Rebeca Ma. Elena Guzmán Saldaña*** \\ Universidad Autónoma del Estado de Hidalgo
}

\section{RESUMEN}

En la actualidad, muchas personas comienzan a cambiar la visión que tienen acerca del matrimonio y optan por la unión libre, la soltería e, incluso, relaciones a corto plazo o a distancia. Con el objetivo de identificar las actitudes hacia estos tópicos en estudiantes de preparatoria de la Universidad Autónoma del Estado de Hidalgo se aplicó la escala de Actitudes hacia la transformación de la vida en pareja de García (2011). Este instrumento está compuesto por siete factores (33 reactivos) que explican el 59.389\% de la varianza, con una consistencia interna de .886. Los participantes fueron 296 adolescentes: 172 mujeres y 124 hombres. Los resultados muestran una mayor aceptación hacia temas relacionados con el matrimonio tardío y la paternidad en soltería. En cambio, aspectos como el sexo premarital obtienen poco apoyo. Los resultados son discutidos en términos de la transformación de la vida en pareja en México.

Palabras clave: actitudes, unión libre, matrimonio, sexualidad, jóvenes.

\section{ABSTRACT}

Nowadays many people begin to change their point of view about marriage and prefer to choose cohabitation, singlehood, and even long-distance relationships. In order to identify attitudes towards these topics, the scale of attitudes towards transformation of married life was administered to high school students from the Autonomous University of Hidalgo State. The scale consists of seven factors (33 items) that explain the 59,389\% of the variance, with an internal consistency of.886. Participants were 296 adolescents, 172 women and 124 men. The results show a greater acceptance issues related to the late marriage and single parenthood. However issues such as premarital sex get little support. The results are discussed in terms of the transformation of couple life in Mexico.

Keywords: attitudes, cohabitation, marriage, sexuality, young people.

* Melissa García Meraz. Universidad Autónoma del Estado de Hidalgo, melissaunam@yahoo.com.mx, 57001702

** Alejandra Salvador García. Universidad Autónoma del Estado de Hidalgo, jja107@hotmail.com, 7717172000 ext. 5104

*** Rebeca Ma. Elena Guzmán Saldaña. Universidad Autónoma del Estado de Hidalgo, remar64@yahoo.com.mx, 717172000 ext. 5104 


\section{INTRODUCCIÓN}

De acuerdo con Morris y DePaulo (2009), es común asumir que la vida en matrimonio aumenta la felicidad y más aún, que a diferencia de los solteros, las personas casadas son más felices. Es decir que, como un acto natural de crecer, del desarrollo personal y del ideal de "alcanzar la felicidad", las personas asumen que después del ejercicio de tener varias parejas, salir en citas e invertir mucho tiempo, dinero y esfuerzo en ellas, el punto culminante es llegar al matrimonio. Con ello se alcanza la meta "ideal" de todas las citas: vivir en pareja y ser feliz. Sin embargo, datos recientes revelan que no existen diferencias en el nivel de felicidad entre las personas que están casadas y aquellas que deciden permanecer solteras (Morris \& DePaulo, 2009).

Más allá del ideal del matrimonio, muchas personas comienzan a cambiar la idea que tienen acerca de éste y prefieren optar por la unión libre, la soltería e incluso, relaciones a distancia o relaciones en las que cada integrante de la pareja tiene una residencia particular pero deciden reunirse los fines de semana. A estas parejas se les llama Living Apart Together, LAT (García \& Martínez, 2010; Trost \& Levin, 1999), término que indica que son parejas estables, marcadas por la fidelidad, el amor y el compromiso, pero que deciden no tener una residencia en común, sino mantenerse fuera del matrimonio pero también fuera de la unión libre.

De hecho, datos del Instituto Nacional de Estadística, Geografía e Informática (2012) muestran que en México, el número de personas que han optado por la unión libre como una opción para vivir en pareja ha aumentado, pasando de un $7.0 \%$ en 1990 , a un $15.7 \%$ en 2012. Los datos referentes a la unión y la disolución marital también muestran un aumento en el número de divorcios y de separaciones, al pasar de un $7.2 \%$ en 1990 a un $15.1 \%$ en 2009 , así como una disminución en el índice de matrimonios en un 21\% (INEGI, 2012). Con ello, se muestra una panorámica en la cual los individuos comienzan a optar por la unión libre, no sólo como una experiencia previa o experimental al matrimonio, sino realmente como una forma de unión y de vida en pareja. Es así que el aumento del divorcio, la soltería, la postergación del matrimonio y la apertura hacia la unión libre muestran que la sociedad mexicana se mueve hacia una nueva configuración de pareja y hacia un nuevo plan de vida.
En México, las instituciones, centros de bienestar social y aún los individuos se encuentran poco preparados para conceptualizar estos cambios. ¿Pueden las personas planear una vida en soltería?, ¿tienen los mismos beneficios los padres solteros y las madres solteras que aquellos que viven en pareja?, o incluso, aquellos que han decidido no casarse ¿pueden planear la maternidad o paternidad sin anteponer primero la vida en pareja? Dadas las estadísticas se prevé que el divorcio, la separación y la soltería aumenten, mientras que el matrimonio disminuirá. Sin embargo, no existen estudios en México que muestren el grado de aceptación o rechazo hacia estos temas por parte de los adolescentes y jóvenes del país. Por ello, el presente artículo muestra datos empíricos acerca de la postura de adolescentes a nivel bachillerato de la ciudad de Pachuca sobre temas relacionados a la soltería, la unión libre, el sexo premarital y extramarital así como la paternidad en soltería.

\section{Transformación de la vida en pareja}

La literatura indica que, en general, las personas han transitado hacia una aceptación de la paternidad fuera del matrimonio, la unión libre, el matrimonio tardío, el divorcio como un medio para no dañar a los hijos, como una solución cuando existen problemas en el matrimonio, así como hacia el sexo premarital. En cambio, aún condenan situaciones como el sexo extramarital (Axinn \& Thornton, 2000; Thornton \& Young DeMarco, 2001).

De igual manera, tanto en México como en el resto del mundo, las mujeres esperan cada vez más para ser madres, lo que posibilita que tengan un desarrollo escolar y profesional que no se había visto antes. Incluso muchas aguardan el éxito profesional antes de iniciar una familia. Datos en México han mostrado que las capitalinas esperan un mayor tiempo entre el primer $y$ el segundo hijo, lo cual muestra una mayor planificación familiar y tiempo dedicado a sus carreras profesionales (Reforma, 2003). Estos aspectos han incidido en el cambio legislativo de algunas entidades como el Distrito Federal, al legalizar el aborto a través de la ILE o Interrupción Legal del Embarazo (GDF, 2012).

Sin embargo, muchos opinan que el matrimonio y la vida en pareja siguen siendo una expectativa de vida para hombres y mujeres jóvenes. Aún más, aquellos 
que ya se han divorciado o separado siguen buscando una y otra vez volverse a enamorar y casar, incrementando el número de matrimonios por segunda vez; de hecho la probabilidad de que un hombre permanezca soltero después de divorciarse es poco común.

En Estados Unidos se estima que 75\% de los hombres se casan nuevamente; en el caso de las mujeres lo hace un 66\% (Brown \& Amatea, 2000). Datos del INEGI reportan que de los hombres de 60 años y más, sólo el $19.1 \%$ permanece soltero, mientras que en el caso de las mujeres es de $46 \%$ (INEGi, 2012). De hecho, después del matrimonio y el divorcio hombres y mujeres siguen buscando la posibilidad de vivir en pareja; esto lo evidencia la cantidad de páginas en internet dedicadas a la concertación de citas. Esto marca la pauta hacia una actitud positiva hacia la vida en pareja y hacia el matrimonio.

De acuerdo con Thornton y Young DeMarco (2001), en la actualidad existe una mayor apertura hacia la unión libre, sobre todo en el caso de las mujeres. En países como Suecia y Dinamarca se popularizó a partir de 1969, mientras que en otros países de Europa se dio a principios de los años setenta (Hall \& White, 1995). Hoy en día, las cifras son tales que en el caso de los Estados Unidos se estima que al menos la mitad de todos los individuos con edades de entre 35 y 39 años han cohabitado en algún momento de sus vidas (Waite \& Gallagher, 2000). En México, las estadísticas indican que comenzó a incrementarse el número de personas cohabitando a partir de los años cincuenta, popularizándose en los sesenta, al llegar a un $15.7 \%$ en 2010 (INEGI, 2007; INEGI, 2012).

\section{Vida en soltería y sexo premarital}

En países como Inglaterra se plantea que para el año 2026, el número de personas solteras será de alrededor del $70 \%$. Es decir, personas que nunca se habrán casado y que probablemente permanezcan así (The times, 2008). Aún con el incremento de quienes viven en soltería ya sea como modo de vida o porque deciden posponer el matrimonio, hay evidencia de que también se da una idea estereotipada acerca de este estado. Comparados con las personas que están casadas se percibe a los solteros como más inmaduros socialmente, egoístas, pobremente ajustados, poco atractivos, o no atractivos, asociales, desagradables y, frecuentemente, con baja autoestima. La única visualización positiva es que son vistos como más independientes y orientados al trabajo (Morris \& DePaulo, 2009).

De acuerdo con Morris y DePaulo (2009) diversas investigaciones han mostrado que los hombres solteros ganan menos dinero y tienen menos promociones que los casados, aun cuando tienen la misma edad, el mismo nivel de estudios, destreza y muestran los mismos resultados en sus ejecuciones laborales. De acuerdo con los autores, se les percibe como más orientados al trabajo y más responsables. Sin embargo, esta percepción también tiene un efecto negativo, ya que a menudo se les pide que trabajen más tiempo, horas extra y durante las vacaciones, aun cuando es menos probable que reciban promociones en comparación con sus compañeros casados y con hijos. Incluso se les puede pedir que trabajen suplantando a estos compañeros. La sociedad discrimina a los solteros independientemente de la situación en la que se presente la soltería. Muchas veces son mal vistos como personas que no han llegado al ideal de pareja; puede percibirse que son menos atractivos, estables, sociables y deseables en comparación con las personas casadas (Morris \& DePaulo, 2009).

A pesar de las críticas dirigidas hacia quienes permanecen solteros, estos son más allegados a sus familias, cuidan a sus padres, quieren más a sus amigos y se preocupan por hacer más estrechas sus relaciones con sus vecinos; asimismo, las posibilidades de realizar más actividades aumentan en esta situación, ya que pueden disponer de su tiempo de la manera que más les sea conveniente y que se ajuste a sus actividades, pueden gozar de una economía más holgada, realizar actividades de esparcimiento con amistades o sin ellas, pasar tiempo con su familia, incluso tener más espacio en su residencia, por ello, se afirma que la soltería tiene sus ventajas (Morris \& DePaulo, 2009).

\section{Matrimonio tardío, paternidad en soltería y sexo extramarital}

La soltería ha jugado un papel importante dentro de la sexualidad. Al posponerse el matrimonio y aumentar el periodo de soltería, la lógica se inclina a aceptar cada vez más el sexo premarital, llevando consigo una apertura hacia la sexualidad fuera del matrimonio. De acuerdo con Houts (2009), entre 1970 y 1990 disminu- 
yó la edad del debut sexual, tanto en hombres como en mujeres. El autor plantea muchas razones para explicar este cambio, entre las que destaca los cambios históricos, tales como la revolución sexual, el incremento de la edad para contraer matrimonio, la soltería y una supervisión parental menos rigurosa, especialmente cuando los padres están divorciados o ambos trabajan fuera del hogar.

Sin embargo, la infidelidad marital, es decir, el sexo extramarital se juzga cada vez más (Axinn \& Thornton, 2000; Thornton \& Young DeMarco, 2001). Así, el ideal de la fidelidad sexual todavía se considera un aspecto más importante de la vida en pareja. De acuerdo con Goodwin (2009), el matrimonio se valora mucho, tanto por la sociedad como por la pareja. Tradicionalmente, el matrimonio tiene el significado de regular la expresión de la sexualidad. La soltería y el sexo premarital están muy vinculados al tema del matrimonio tardío. Hombres y mujeres esperan cada vez más tiempo para casarse. Muchas veces el éxito profesional y la educación universitaria permiten que la persona posponga el matrimonio.

\section{MÉTODO}

Con el objetivo de conocer las actitudes acerca de la unión libre, la soltería, la paternidad en soltería, así como el sexo premarital y extramarital se aplicó el instrumento de Transformación de la vida en Pareja de García (2011). En un segundo análisis se realizaron pruebas estadísticas para conocer las diferencias entre hombres y mujeres, a través de la prueba t de Student.

\section{Participantes}

Se trabajó con un grupo de participantes seleccionados por conveniencia, tomando en cuenta los grupos de primero, tercer y quinto semestre de la Preparatoria número 3 de la Universidad Autónoma del Estado de Hidalgo. En total, la muestra quedó constituida por 296 adolescentes: 172 mujeres y 124 hombres. Los participantes reportaron un rango de edad de 15 a 19 años, con una media de 16.98 años. Todos dijeron ser solteros.

\section{Instrumento}

El instrumento de Transformación de la vida en Pareja de García (2011) fue validado en una muestra de 500 pobladores del estado de Hidalgo, estudiantes de preparatoria y licenciatura. Está conformado por siete factores: 1) Apertura hacia la unión libre (siete reactivos), 2) Sexo premarital (cinco reactivos), 3) Sexo extramarital (cinco reactivos), 4) Apertura marital (cinco reactivos), 5) Vida en soltería (cuatro reactivos), 6) Matrimonio tardío (cuatro reactivos), 7) Paternidad en soltería (tres reactivos). Los siete factores explican el $59.389 \%$ de la varianza, una consistencia interna de .886. En total, el instrumento está conformado por 33 reactivos.

El formato de respuesta es una escala Likert que va de 1 "completamente en desacuerdo" a 5 "completamente de acuerdo". La tabla 1 muestra el nombre de los siete factores y algunos ejemplos de la escala.

Tabla 1. Reactivos de ejemplo de la escala de Transformación de la vida en pareja, porcentaje de varianza explicada y alfa de Cronbach

\begin{tabular}{|c|c|c|}
\hline FACTORES & \%V.E. & Alfa \\
\hline 1.Apertura hacía la unión libre & & \\
\hline La unión libre es la mejor opción para vivir e & \multirow{3}{*}{$26.03 \%$} & \multirow{3}{*}{.841} \\
\hline Es preferible vivir en unión libre que casarse & & \\
\hline Vivir en unión libre es lo de hoy & & \\
\hline
\end{tabular}




\begin{tabular}{|c|c|c|}
\hline FACTORES & $\%$ V.E. & Alfa \\
\hline 2. Sexo premarital & & \\
\hline Es mejor tener relaciones sexuales hasta el matrimonio & \multirow{3}{*}{$8.80 \%$} & \multirow{3}{*}{.514} \\
\hline Es adecuado que una persona joven experimente su sexualidad antes de casarse & & \\
\hline Está bien que un hombre tenga relaciones sexuales antes del matrimonio & & \\
\hline 3. Sexo extramarital & \multirow{3}{*}{$7.64 \%$} & \multirow{3}{*}{.783} \\
\hline $\begin{array}{l}\text { Una mujer casada puede experimentar sexualmente con otras personas además de su } \\
\text { marido }\end{array}$ & & \\
\hline $\begin{array}{l}\text { Está bien que una persona experimente sexualmente fuera de su matrimonio con otras } \\
\text { personas }\end{array}$ & & \\
\hline 4. Apertura marital & \multirow{3}{*}{$5.50 \%$} & \multirow{3}{*}{.708} \\
\hline Está bien que una persona decida nunca casarse y vivir sola & & \\
\hline Es aceptable que una pareja decida no tener hijos & & \\
\hline 5. Vida en soltería & \multirow{3}{*}{$4.38 \%$} & \multirow{3}{*}{.688} \\
\hline Hoy en día es preferible vivir solo que con una pareja & & \\
\hline Los jóvenes hoy en día prefieren vivir solos a casarse & & \\
\hline 6. Matrimonio tardío & \multirow{3}{*}{$3.74 \%$} & \multirow{3}{*}{.538} \\
\hline Está bien que una persona se case hasta que tenga un trabajo estable & & \\
\hline Es mejor casarse cuando ya se ha terminado de estudiar & & \\
\hline 7. Paternidad en soltería & \multirow{3}{*}{$3.28 \%$} & \multirow{3}{*}{.723} \\
\hline Está bien que un hombre que no tiene pareja decida tener hijos & & \\
\hline Una mujer que no tiene pareja puede educar bien a sus hijos & & \\
\hline
\end{tabular}


En la última sección del instrumento se les preguntó por su edad, sexo, nivel de estudios y estatus de pareja. Además, se incluyó un apartado con las siguientes preguntas:

Tabla 2. Preguntas del Instrumento de Actitudes hacia la vida en pareja

1. ¿Te gustaría casarte algún día?

2. En los próximos cinco años ¿Qué piensas hacer?
a) Casarte
b) Tener un hijo
c) Vivir en unión libre
d) Permanecer soltero
e) No sé

3. ¿Cuál es la edad ideal para casarse?
$15-18 \quad 19-22 \quad 23-26 \quad 27-30$
31-34 35 o más No sé

\section{Procedimiento}

La aplicación se llevó a cabo en grupos dentro de las instalaciones preparatorias de la Universidad Autónoma del Estado de Hidalgo. Se le aseguró a cada estudiante la confidencialidad de sus respuestas. Los datos fueron procesados con el paquete estadístico SPSS versión 15 para Windows.

\section{RESULTADOS}

La imagen 1 muestra los puntajes obtenidos para los siete factores de la escala. El puntaje más alto y de mayor acuerdo para la población es el relacionado a Matrimonio Tardío (4.2), mientras que el más bajo es el factor de sexo extramarital (1.71).

\section{Diferencias por sexo}

Un segundo análisis permitió identificar las diferencias entre hombres y mujeres. Los resultados muestran que aquellos factores relacionados al sexo premarital, extramarital y la paternidad en soltería son los únicos que obtienen puntajes diferenciales entre hombres y mujeres.

\section{Imagen 1. Medias para cada factor de la escala de Transformación de la vida en pareja}

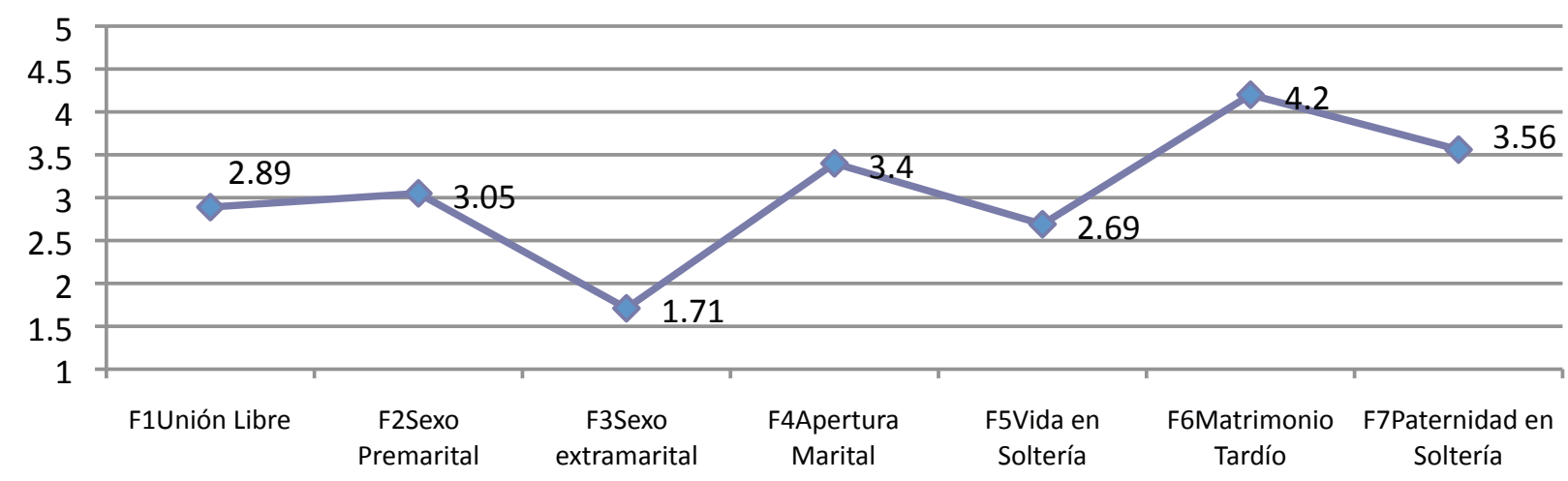


Tabla 3. Medias de los factores sexo premarital, extramarital y paternidad en soltería con la significancia de la prueba Levens así como la prueba t.

\begin{tabular}{|l|c|c|c|c|c|c|}
\hline Factor & Hombres & Mujeres & Levens & t Student & g. I. & Sig. \\
\hline Sexo premarital & 3.31 & 2.85 & .439 & -4.412 & 294 & .000 \\
\hline Sexo extramarital & 1.94 & 1.52 & .007 & -4.558 & 222.090 & .000 \\
\hline Paternidad en soltería & 3.42 & 3.66 & .044 & 2.196 & 236.41 & .031 \\
\hline
\end{tabular}

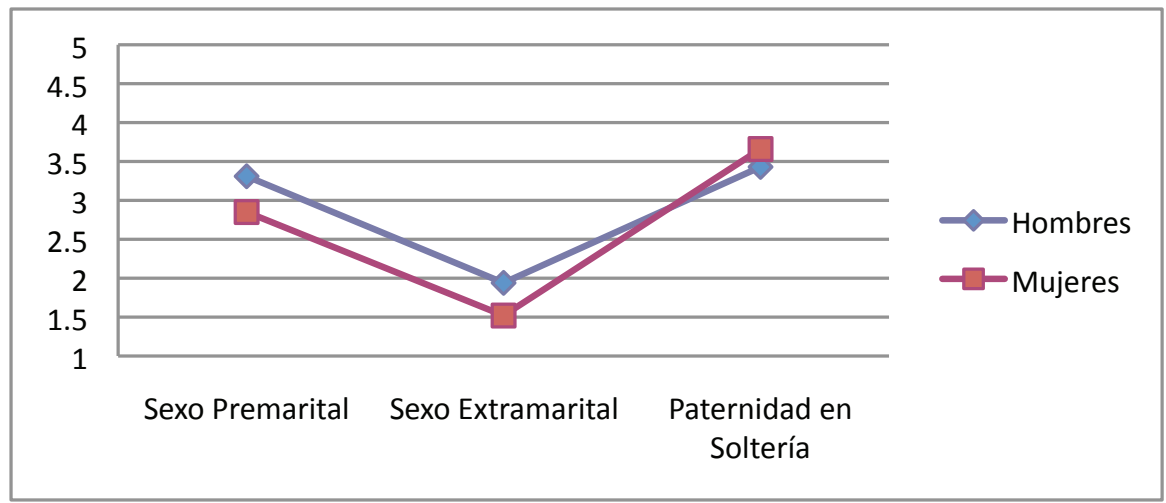

Imagen 2. Medias de hombres y mujeres para los factores que mostraron diferencias

Las diferencias muestran que en el caso de las mujeres obtienen puntajes más bajos en comparación con los hombres en aquellos factores relacionados a la sexualidad. Mientras que los hombres obtienen puntajes más bajos en el factor relacionado a la paternidad en soltería.

Con referencia a las últimas preguntas, los datos muestran que el $88 \%$ (183 participantes) contestaron que desearían casarse algún día contra un $12 \%$ que reportó que no le gustaría casarse (26 participantes).
En los próximos cinco años la mayoría de los participantes planea permanecer soltero. Sólo un 12\% planteó casarse y $9 \%$ vivir en unión libre.

Finalmente, 130 participantes opinan que la edad preferible para casarse es de los 23 a 26 años, seguidos de 125 participantes que plantean que la mejor edad para casarse es entre los 27 y 30 año de edad.

Tabla 4 Frecuencia de respuesta de la pregunta ¿Cuál es la edad ideal para casarse?

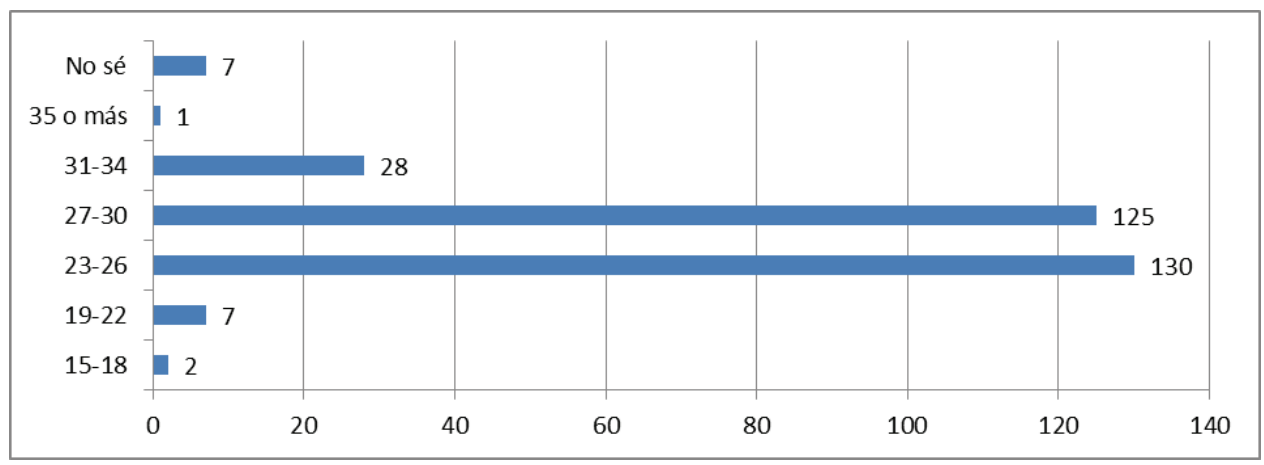




\section{DISCUSIÓN}

Los resultados muestran que, en términos generales, existe una mayor apertura hacia el matrimonio tardío (4.2), y una aceptación hacia la paternidad en soltería (3.56). Estos datos indican que los jóvenes visualizan una actitud positiva hacia una mayor espera para contraer matrimonio, de igual manera, el educar a un hijo fuera del matrimonio es bien visto.

Es así que se muestran dos posturas fundamentales; existe una aceptación clara hacia casarse después de que se ha terminado de estudiar o se tiene éxito profesional, así como la paternidad fuera del matrimonio. Ambos evidencian una constante en el caso de los jóvenes estudiantes que visualizan la vida en matrimonio como algo que puede postergarse. Sin embargo, con la apertura de la sexualidad, también les queda claro que puede darse la paternidad en soltería y que ésta es aceptable, y puede ser tan certera como la que se comparte entre padre y madre. Estos resultados concuerdan con lo que se ha reportado en la literatura (Axinn \& Thornton, 2000; Thornton \& Young DeMarco, 2001).

Sin embargo, lo cierto es que el ideal del matrimonio se mantiene, ya que muchos jóvenes visualizan su postergación pero no su ausencia. Los jóvenes esperan casarse y no ven la vida en soltería como algo tan bueno (media 2.69). La idea de "es mejor vivir sólo que mal acompañado", es decir, reactivos como "Hoy en día es preferible vivir solo que con una pareja”, y "Los jóvenes hoy en día prefieren vivir solos que casarse" no son tan apoyados (pertenecen al factor 3 Vida en soltería). En cambio sí lo es la idea de buscar con quién vivir bien (Matrimonio tardío 4.2). Lo mismo sucede con el sexo extramarital (1.71).

Sobresale el hecho de que la vida en soltería no alcanza siquiera la media teórica (2.69). Quizás se deba en mucho al estereotipo social que se tiene acerca de las personas que deciden vivir solas. En muchos contextos se les describe como "solteronas" "quedadas" y "amargadas". Aunado a otras expresiones de la vida cultural mexicana que preguntan, “¿Cómo es posible que viva solo?” “QQuién lo va a cuidar?”. De hecho la literatura muestra que los hombres que se divorcian son más proclives a volverse a casar; muchos resultados indican que es porque buscan a alguien que los cuide. En Estados Unidos, los hombres que permanecen solteros después del divorcio viven menos y su calidad de vida es menor a la de sus pares, mientras que muchas mujeres pueden optar por el rematrimonio como una forma de recuperar la economía, por lo que buscan parejas con una posición económica estable (Brown \& Amatea, 2000). Esto se debe a que las mujeres solteras tienen un círculo de apoyo más amplio, incluidas las amigas, comadres, vecinas, familia, e incluso la familia de su ex esposo (García \& Martínez, 2010). Aunado a ello, la sociedad discrimina a los solteros independientemente de la situación en la que se presente la soltería (Morris \& DePaulo, 2009). Sin embargo, y cómo se comentó anteriormente, la soltería conlleva también una parte positiva, que se refleja en tiempo dedicado a uno mismo, postergación de la maternidad o paternidad, tener más cercanía con los amigos y alcanzar un desarrollo profesional pleno (Morris \& DePaulo, 2009).

En el caso de las actitudes hacia la unión libre apenas reflejan un puntaje sobre la media teórica (3.4), lo cual revela una incipiente aceptación hacia esta situación como una alternativa al matrimonio y no sólo como un ensayo hacia éste. De acuerdo con las estadísticas, es claro que en el futuro las sociedades estarán en mayor medida marcadas por la soltería, el matrimonio tardío, la unión libre y las relaciones Living Apart Together (DeJong, 2002). En la sociedad moderna, las relaciones comenzarán cada vez más a definirse en base a la afinidad (cariño, amistad, similitud) y menos por la consanguineidad (padres, hijos). Este tipo de transformaciones en las relaciones cercanas marca una necesidad creciente de información y educación en diversos tópicos. Si las personas postergarán el matrimonio y al mismo tiempo tendrán una mayor apertura hacia la sexualidad, entonces será necesaria una mayor información acerca de estos temas e, incluso, a edades más tempranas. Además, será necesaria una educación sexual más amplia y más libre, sacando a la sexualidad del contexto del matrimonio, pero también de la vida privada, y llevándola a la discusión en la esfera pública. Aún, cuando al parecer nuestro país todavía se encuentra en este tránsito entre la aceptación de la unión libre y hacia la clara tendencia a la cohabitación como alternativa total al matrimonio.

Otro de los cambios que se han dado en la esfera social es que cada vez más mujeres trabajan fuera del hogar. Deciden en dónde y con quién vivir, aunque a veces eso implique quedarse en la casa paterna. A dife- 
rencia de otros países, en donde las personas deciden vivir en relaciones Living Apart Together para mantener su independencia, es decir, cada quién su casa y mantenemos una relación romántica, en México muchas relaciones LAT se dan por la falta de recursos económicos, así cada quien permanece en la casa de sus padres (García \& Martínez, 2010). A esto se suman las faltas de oportunidades económicas y la pobreza del país. Hombres y mujeres se quedan a vivir en la casa de sus padres, ya que la economía no les favorece para su subsidio.

La soltería, así, parece no ser una opción "permanente" sino un estado que puede alargarse lo suficiente y que, una vez obtenidos el éxito personal y profesional puede concretarse en matrimonio o unión libre. La poca aceptación hacia la soltería podría deberse a los estereotipos que están ligados a ella (Morris \& DePaulo, 2009), a pesar de que son personas mucho más cercanas a sus padres y a sus amigos. ¿Qué se necesita entonces para visualizar la soltería de manera positiva? ¿Es necesario ser soltero para visualizarla positivamente? $\mathrm{O}$ tendremos que acostumbrarnos a ver a las personas como "solteronas" "quedadas" y "amargadas".

Las instituciones que promueven la vida en matrimonio, difunden también una idealización de éste, lo que a nivel legal se traduce en discriminación, ya que contraer nupcias permite adquirir una casa, ejercer créditos, tener avales, poder adoptar; incluso formar parte de la seguridad social, etc. La sociedad y las instituciones no están preparadas para las madres y padres solos; mucho menos para los solteros, todo se ha construido y preparado para las familias, y a pesar de que ahora el número de éstas sea menor, aún sigue siendo una sociedad para la familia, no para los solteros y aquellos que deciden vivir solos. Sin embargo, concepto con respecto a la soltería necesita cambiar, ya que los solteros, ya sea porque así han decidido su estilo de vida, ya sea porque deciden casarse a edades muy tardías, o simplemente porque ya se han divorciado y deciden permanecer así, necesitan una nueva visualización de su estilo de vida.

Las diferencias entre los grupos de hombres y mujeres también tienen un papel interesante, ya que, de acuerdo con los patrones de género, las mujeres están en mayor medida de acuerdo con temas como la paternidad en soltería. Esto se debe, en gran parte, a que muchas mujeres deciden tener hijos fuera del matrimonio; de hecho, los hogares dirigidos por mujeres en nuestro país no son nuevos, han estado presentes a lo largo de toda su historia.

En temas relacionados con la sexualidad existe también una clara diferencia de género, ya que con referencia al sexo premarital, los hombres mantienen una actitud más positiva que las mujeres. Incluso los hombres tiene respuestas más positivas hacia el sexo premarital, permisividad sexual, masturbación y sexo casual (Petersen \& Hyde, 2010).

De esta manera, las actitudes hacia la transformación de la vida en pareja no son uniformes en todos los temas. Existe una apertura más clara hacia la paternidad y la postergación del matrimonio, así lo evidencian las preguntas libres que se realizaron. El 70\% indica que sus planes en los próximos años serán permanecer solteros. De acuerdo con los participantes, la mejor edad para casarse va de los 23 a los 30 años. Es así que chicos y chicas siguen visualizando el matrimonio como una parte fundamental de su plan de vida; sin embargo, éste puede postergarse a favor de otros planes de vida. Sin duda, las instituciones deberán contemplar este tipo de situaciones para garantizar el bienestar de hombres y mujeres en transformación de la intimidad y la vida en pareja.

\section{CONCLUSIONES}

Existen diversas limitaciones en el estudio, por ejemplo, el tamaño de la población y la focalización hacia el grupo de preparatorianos. Sin embargo, es destacable porque en México casi no existen estudios referentes a estos temas, aun cuando en Estados Unidos llevan investigándose desde hace más de una década. ¿Estarán las instituciones y la sociedad preparada para los cambios en la estructura familiar?, ¿para la innovación de la vida en soltería y el matrimonio tardío? • 


\section{REFERENCIAS}

Axinn, W. G. \& Thornton, A. (2000). The transformation in the meaning of marriage. En L. J. Waite (Ed.), The ties that bind. Perspectives on Marriage and Cohabitation. New York: Aldine de Gruyter.

Brown, N. M. \& Amatea, E. S. (2000). Love and Intimate relationships. Journeys of the hearth. USA, UK: Taylor \& Francis.

DeJong, G. J. (2002). The dilemma of repartnering: considerations of older men and women entering new intimate relationships in later life. Ageing International, 27(4), 61-78.

García, S. A. (2011). Actitudes hacia la transformación de la vida en pareja: unión libre, soltería, matrimonio tardío, sexo premarital y paternidad fuera del matrimonio. Tesis de licenciatura no publicada. Pachuca, Hidalgo: Universidad Autónoma del Estado de Hildago.

García, M. M. \& Martínez, M. J. P. (2010). Parejas Living Apart Together (LAT) en México, Revista de Psicología Social y Personalidad, 25(1) 49-68.

Gobierno del Distrito Federal, (2012). Recuperado 26/01/2012, de la fuente http://www.df.gob.mx/

Goodwin, R. (2009). Marriage, Historical and CrossCultural Trends, en Harry T. Reis. \& Susan Sprecher (Eds.), Encyclopedia of Human Relationships. Vol.2. USA: SAGE Reference Publications.

Hall, R. \& White, P. (1995). Europe's population: toward the next century. Routledge.

Heaton, T. B. \& Forste, R. (2007). Informal unions in Mexico and the United States. Journal of Comparative Family Studies, 38(1), 55-69.

Houts, P. L. (2009). Sexual Intercourse First Experience. En Harry T. Reis. \& Susan Sprecher (Eds.), Encyclopedia of Human Relationships. Vol.2. USA: SAGE Reference Publications.

INEGI (2012). Estadísticas a propósito del 14 de febrero: Matrimonios y divorcios. Recuperado el 20 de mayo de 2012 http://www.inegi.org.mx/inegi/contenidos/ espanol/prensa/contenidos/estadisticas/2012/matrimonios12.asp? s $=$ inegi $\& \mathrm{c}=2827 \& \mathrm{ep}=83$

INEGI (2007). 14 de febrero, matrimonios y divorcios en México. Recuperado el 14 de febrero de 2010. http:// cuentame.inegi.org. $m x /$ poblacion/myd.aspx?tema

Morris L. W. \& DePaulo M. B. (2009) Singlehood. En Harry T. Reis \& Susan Sprecher Encyclopedia of Human Relationships. Vol.2. USA: SAGE Reference Publications.
Petersen, J. \& Hyde, J. S. (2010). Gender Differences in Sexuality. En Joan C. Chrisler \& Donald R. McCreary (Eds). Handbook of Gender Research in Psychology, Volume 1. USA: Springer.

Reforma (2003). Posponen capitalinas la maternidad. Jueves 6 de noviembre de 2003. Anayansin Inzunza.

Thornton, A. \& Young-DeMarco, L. (2001). Four Decades of Trends in Attitudes Toward Family. Issues in the United States: The 1960's Through the 1990's. Journal of Marriage and the Family, 63, 1009-1037.

Times, the (2008). The middle-aged, middle-class couples living 'apart together' who add to housing shortage, Nov 19, p. 21.

Trost, J. \& Levin, I. (1999). Parejas sin domesticidad común. Desacatos Revista de Antropología Social, 2, 73-86.

Waite, L. \& Gallagher, M. (2000). The case for marriage: Why married people are happier, healthier, and better off financially. New York: Double Day.

Inhelder, B. \& Piaget, J. (1996). De la lógica del niño a la lógica del adolescente: Ensayo sobre la construcción de las estructuras operatorias formales. Barcelona: Paidós.

Roche Olivar, R. (2006). Psicología de la pareja y de la familia: Análisis y optimización. Barcelona: Servei de Publicacions Universitat Autónoma de Barcelona.

Santrock, J. (2004). Adolescencia. Madrid: McGraw Hill.

Valdez, J. L. (1991). Las categorías semánticas, usos y aplicaciones en psicología social. (Tesis de maestría inédita). UNAM: México.

Valdez, J. L. (2002). Las redes semánticas naturales, usos y aplicaciones en la psicología social. Toluca: Universidad Autónoma del Estado de México.

Zermeño, A. I., Arellano, A. C. \& Ramírez, V. A. (2005). Redes semánticas naturales: Técnica para representar los significados que los jóvenes tienen sobre televisión, internet y expectativas de vida. (Tesis de licenciatura). Universidad de Colima: Colima.

\section{NOTA DEL AUTOR}

Esta investigación fue realizada en la Facultad de Psicología de la U.M.S.N.H., bajo la asesoría de la Mtra. Dámaris Díaz Barajas.

Fecha de recepción: septiembre 2012 Fecha de aceptación: noviembre 2012 